\title{
Piano Press: A Cable-Actuated Glove for Assistive Piano Playing
}

\author{
Varun Pal', Suhana Shrivastava ${ }^{2}$, and Jaaron Leibson ${ }^{3}$, Yin Yü, Lina Kim, Alanna \\ Bartolini\#, and Diarmid Flatley\# \\ ${ }^{1}$ Bellarmine College Preparatory, San Jose, CA, USA \\ ${ }^{2}$ Mission San Jose High School, Fremont, CA, USA \\ ${ }^{3}$ Tilden Preparatory School, Albany, CA, USA \\ \#Advisor
}

\section{$\underline{\text { ABSTRACT }}$}

As the need for physical therapists for rheumatoid arthritis is rising, novel technologies are emerging to aid in physical therapy and motor recovery for rheumatoid arthritis patients. However, many of the existing techniques pose questions of practicality and use in the real world as multiple patients report being distracted or bored by their physical therapy sessions. To combat this problem, our project Piano Press aims to create a smart wearable glove that combines musical and physical therapy through assistive piano playing. The glove can amplify a user's force when pressing on a piano key and help arthritic pianists develop muscle memory. We built Piano Press with three main modules, the conversion from sheet music to MIDI files and fingering positions on a virtual piano, the overall glove design and the rings around the hand, and the hardware components such as the microcontroller and servo motors. We simulated the finger movement in Rhino 7 with Grasshopper and compared the actuation angle and distance to an experienced piano player's. By utilizing cable actuation and MIDI files to control finger movement, the piano glove would use music to ease symptoms of arthritis while making physical therapy exercises enjoyable.
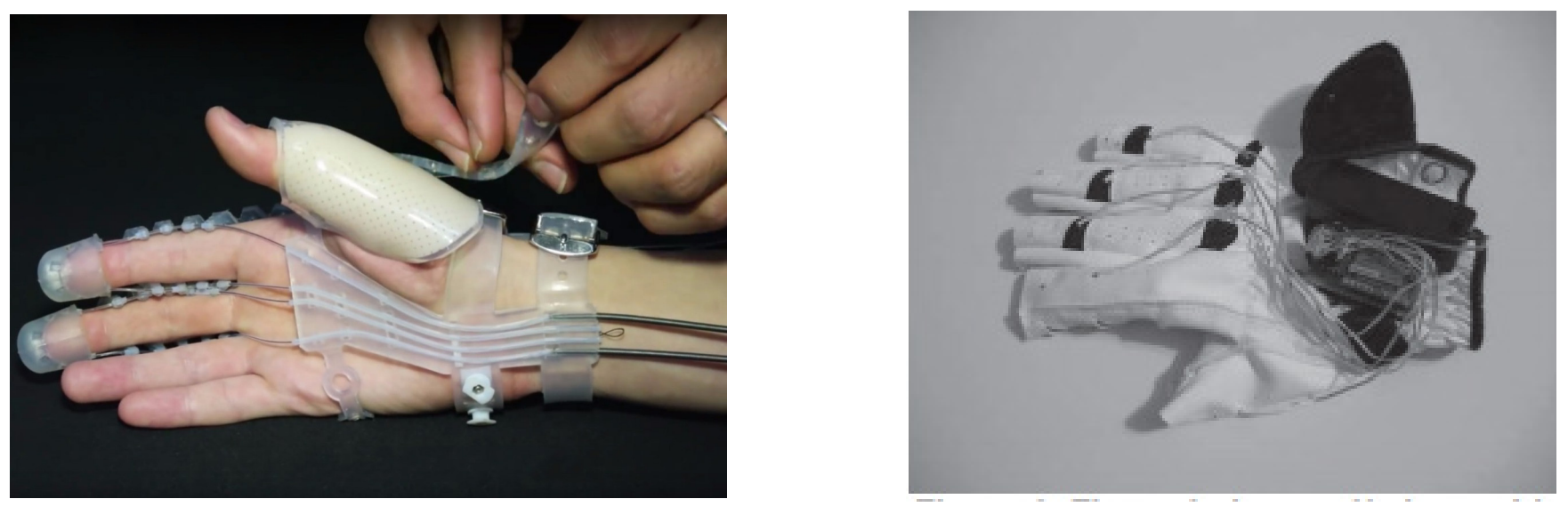

Figure 1: Two previous models of soft robotic gloves to assist patients with the movement of their fingers. The left depicts the Exo-Glove by In et al. and the right depicts PianoTouch by Huang et al. 


\section{Journal of Student Research}

\section{Background}

Rheumatoid arthritis (RA) is a chronic disease that causes disability in the joints and decreased happiness (Smolen et al., 2016). Conventional treatment methods include hand and finger exercises to improve flexibility and reduce pain, such as touching objects or practicing thumb crosses, but these are often tedious and repetitive (Jiang, personal interview, July 13, 2021). Thus, many experimental methods of physical therapy are emerging to engage patients and improve their experiences with daily exercise. One of the more recent technologies that does so is the use of soft robotic gloves that assist patients with finger movement. Previous research regarding hand assistive gloves has been explored and are available in the market, but those devices are mainly used for larger hand motions such as grasping a doorknob (Polygerinos et al., 2015). Pneumatic actuation has been a popular method in such gloves as they can produce very strong forces which can often be too powerful. Moreover, a significant limitation is that pneumatic actuation struggles with speed and precision when moving patients' fingers. This research aims to tackle these limitations and others by creating a cable-actuated glove that targets key exercises used in physical therapy and assists in joint recovery through playing the piano. Piano Press differs from previous cable actuated gloves through its high utility, having the ability to move all 10 fingers, increased mobility, with a mobile hardware pack, and novel software, converting music theory fingering to motor movements.

\section{Methods}

The Piano Press consists of three main modules: the conversion from sheet music to MIDI files and fingering positions on the piano, the overall glove design and the rings around the hand, and the hardware components such as the microcontroller and servo motors. Each module is discussed in further detail below, and an overview of the control process may be found in Figure 2 which incorporates both the software portion (shown in red) and the hardware portion (shown in yellow). 


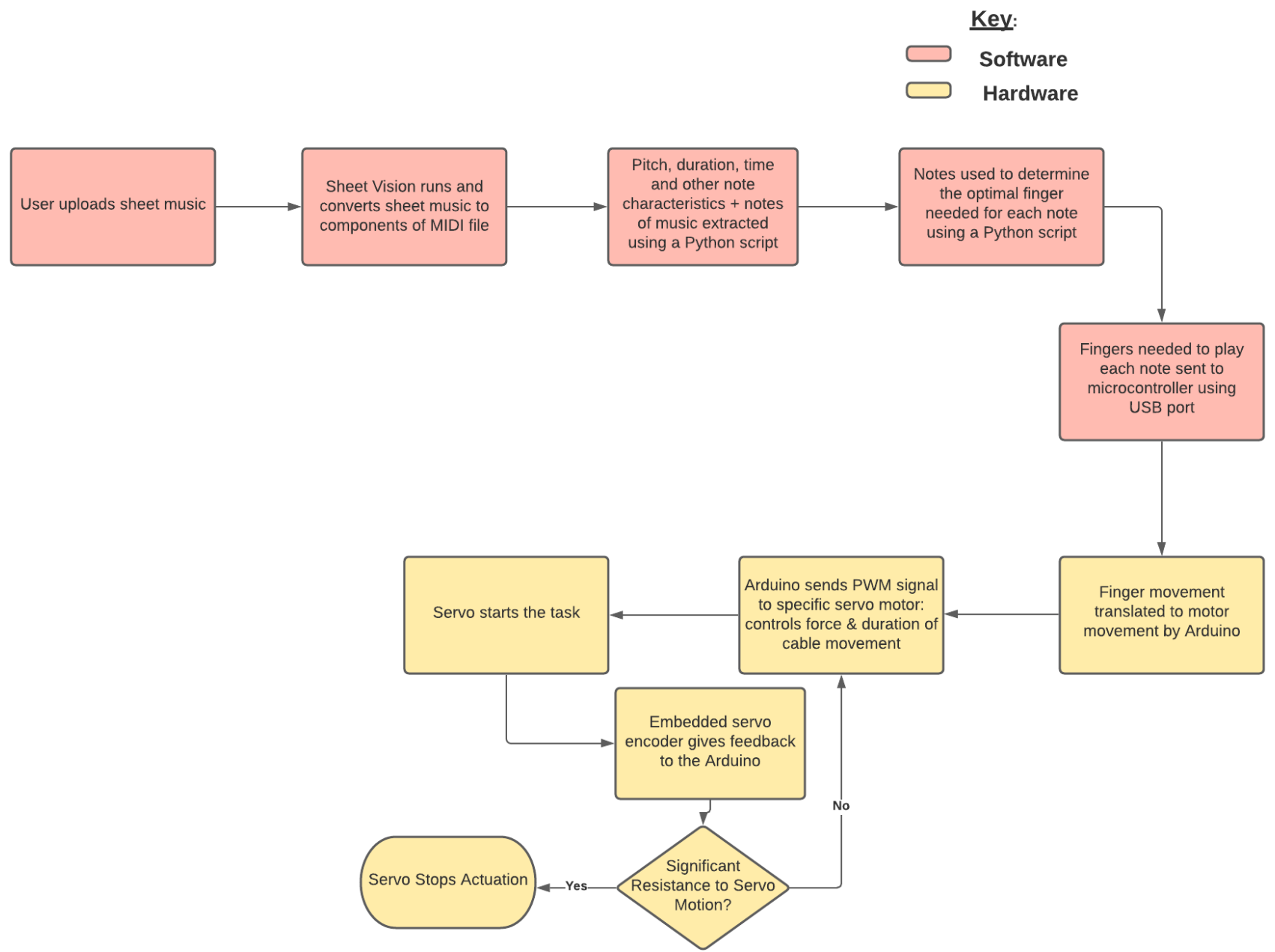

Figure 2: A control diagram for the project.

\section{Modeling the Glove}

A large part of our design considerations were based on the user's comfort. The most important part was to manipulate the user's finger from spots that would cause the least discomfort. Our original design used a total of three mounting spots on the fingers to manipulate the user's hand.
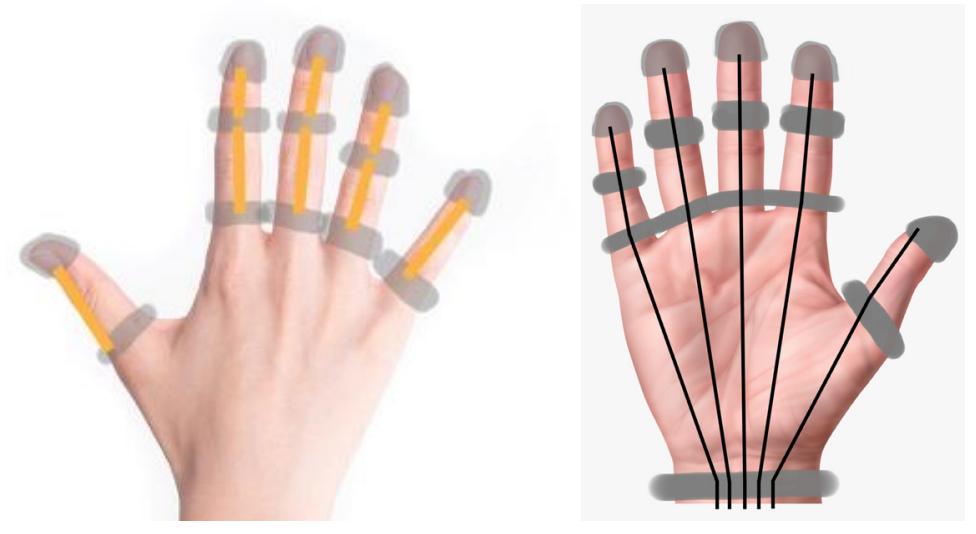
Figure 3: Top view of the glove attached to the user's hand.
Figure 4: Underside view of the glove attached to the user's hand.

This design fully enclosed the user's fingertips in a silicone fingertip to maintain firm control over the fingers. However, this would prevent the user from feeling the keys directly. In addition, fingers do not naturally curve from the fingertip to the knuckles downward, but upward due to the pressure exerted on the key. Using this original design, the user would be put in much discomfort due to being forced to use exclusively their fingertips on the keys. The next design is attached to the fingers at two control points, with a third being mounted around the palm of the hand.

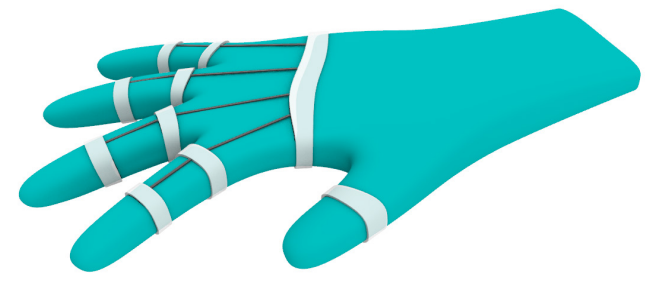

Figure 5: Top view of the second prototype on the user's hand.

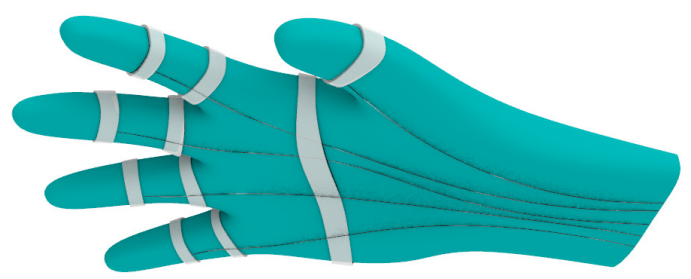

Figure 6: Underside view of the second prototype on the user's hand.

Pulling the fingers from these two control points allows the finger to bend up in a more natural upward curve as opposed to the artificial downward one. The third control point allows for the retraction of the fingers and for the routing of cables to prevent any rubbing on the underside of the hand.

Piano Press is made out of a silicone rubber with a hardness of Shore 30 that allows the glove to be easily slipped onto the user's hand. Silicone maintains a good grip on the user's skin while not creating discomfort on the contact points. Its flexibility allows it to be fit to many different hand shapes and sizes while still maintaining structural integrity. Each finger is actuated through a bowden cable. These cables are enclosed in a PTFE (Polytetrafluoroethylene) tube that prevents the cable from directly rubbing on the skin. PTFE is ideal due to its low friction, allowing the cable to slide along inside it without much resistance. Each one of these is individually routed to the control box where they are pulled to close the fingers.

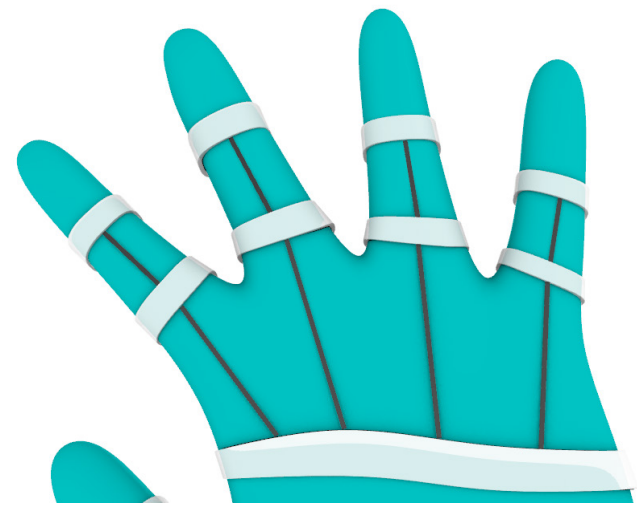

Figure 7: Close up of elastic attaching the control points

To reopen the fingers, the control points are attached by elastic to each other. This elastic pulls the fingers back when slack is put into the cable. Elastic does not generate a high force when stretched, which prevents the finger from snapping back at a high speed. However, the force is great enough to pull any slack through the PTFE tubes to fully open the fingers. 


\section{Piano Pack Components}

In addition to the glove component, the control box is a critical part of the design in actuating the fingers with servo motors. We decided to use our specific type of servo for a few reasons. We chose servo motors instead of standard DC motors or stepper motors because it came with integrated encoders which would help conserve wiring space. Going into the specific servo type among the micro, regular, and jumbo servo sizes to select from, we chose the regular version $(20 \mathrm{~mm} * 40.3 \mathrm{~mm} * 27 \mathrm{~mm})$ because it offered a balance between size and torque. Our benchmark for the force of each servo module's output was from $8 \mathrm{~N}$ to $50 \mathrm{~N}$ which produces sounds from piano to fortissimo respectively or soft to very loud (Kinoshita, et.al, 2007). Additionally, our target for speed was $150 \mathrm{bpm}$ for every quarter note since we estimated this value to be a decent tempo for beginner to intermediate songs. To test each servo type based on our requirements, we used the JVN (John V. Neun) Linear Mechanism Design Calculator. We thus determined that the regular servo motors could pull a $5 \mathrm{lb}$ weight 1 inch (average piano key actuation distance) in 0.3 seconds. This would yield $25 \mathrm{~N}$ of force, creating a forte (loud) sound, played at $180 \mathrm{bpm}$.

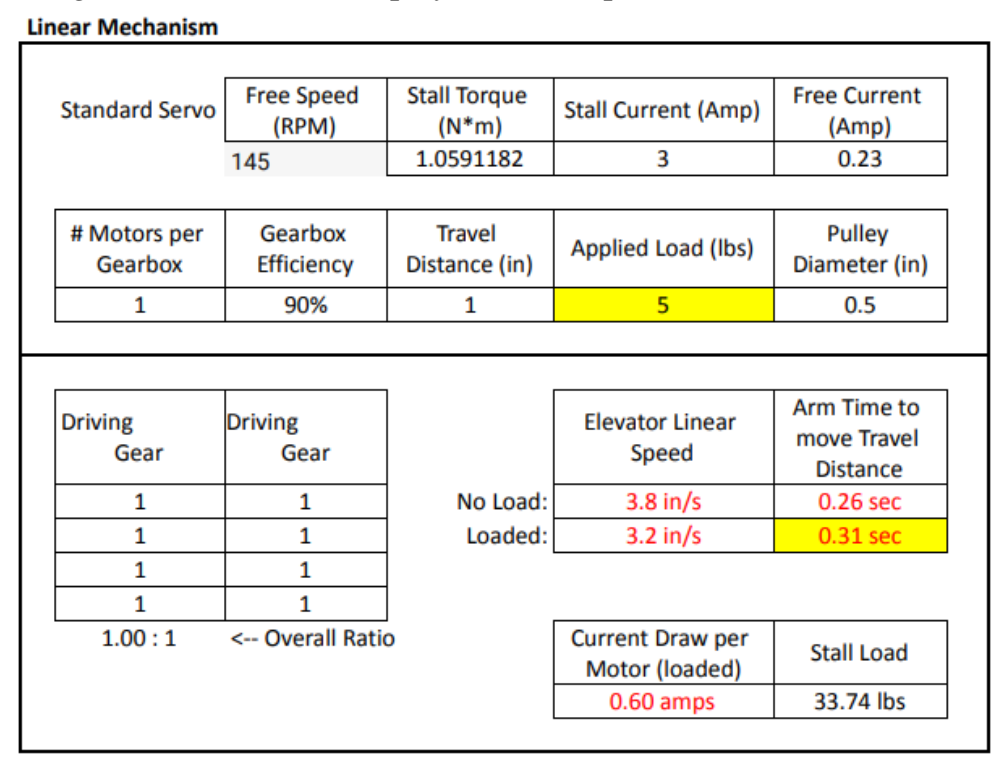

Figure 8: JVN Motor Calculator used to determine speed and force of the different motor types

Another major design factor was the physical location of the control box. We identified two practical mounting locations: on the back and externally on the ground. We ultimately chose to mount the control box on the user's back because it enables the user to easily transport the glove which opens the possibility for more applications of our device in settings other than assistive piano playing. The control box itself contains five motors, one per finger, Bowden cables, and Nylon 101 3D printed spool on each servo. Since the control box is wearable, the back plate of the control box is contoured to a human shoulder blade. In addition to the shape of the box, the use of Bowden Cables was just as important because it allowed us to transmit force from the control box to the glove while maintaining a cable path and reducing friction. Bowden cables consist of an inner metal wire surrounded by a PTFE plastic covering to reduce friction. The metal wire was used to provide rigidity and more tension for the cable path. 


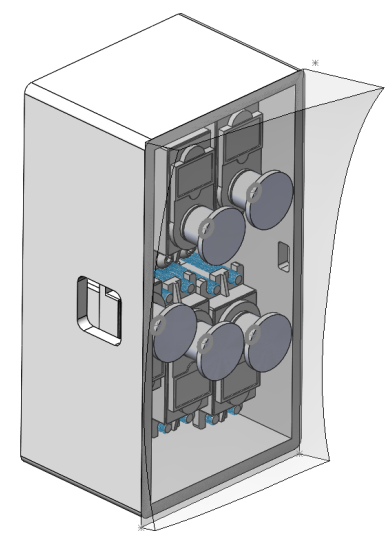

Figure 9: Internals of motor box

The electronics layout, located in between the two motor boxes, consists of an Arduino Mega, PWM and power wires, and eight 9-volt batteries. These components are mounted on a 3D-printed electronics board. Additionally, the electronics mounting board serves as a connector between the two motor modules on each shoulder. The actual logic of the electronics functions is as follows: the Arduino receives finger position data from an external source using the USB port. Next, the Arduino runs a program that translates the finger movement data into motor movement. The Arduino sends a PWM signal to a specific servo motor that controls the force and duration of the cable movement. Then, an embedded servo encoder gives feedback to the Arduino until the actuation is complete. At that point, the PWM signal is stopped and the finger returns to the normal position with the band's elastic pulling force.

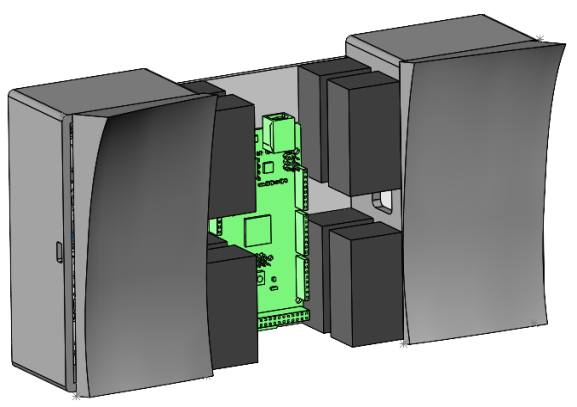

Figure 10: Hardware pack interior

To combine the electronic board and motor modules into a wearable, the Piano Pack was designed to store all the components. The pack can be worn like a backpack and is made out of composite fabric to ensure the user's comfort. 
Figure 11: Hardware pack exterior

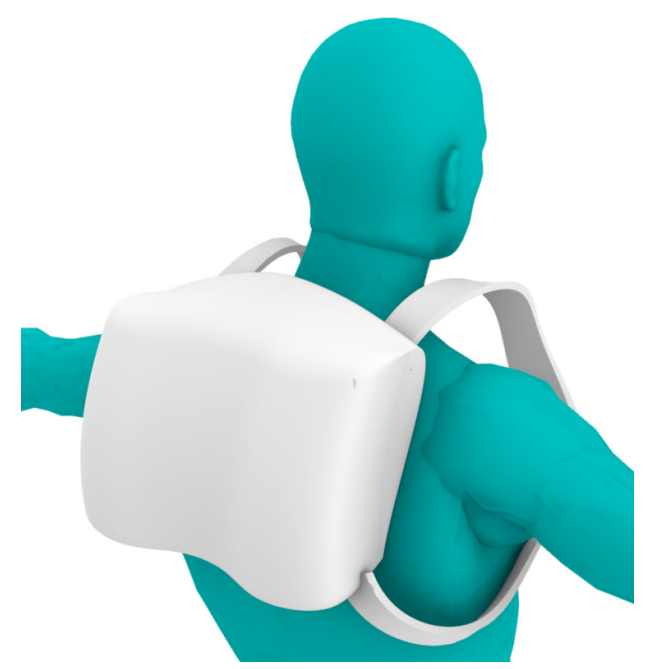

\section{Retrieving and Analyzing MIDI Files}

Our primary approach to determining when pressure should be applied to each finger to bend it requires knowledge of the song users want to play and the notes, pacing, duration of notes, and many other factors required for the song. To utilize an automated, universal method of understanding this, we decided to have the users upload pictures of the sheet music they would like to play and the program would convert the sheet music into MIDI (Musical Instrument Digital Interface) files. While they are binary files and thus only computer-readable, MIDI files can hold a plethora of necessary information about notes inside of songs such as the duration of the note, magnitude of sound, and the time the note starts. These files are widely used when projects use external components due to their compact size and ability to be sent over low-bandwidth connections. For these reasons, we chose to employ a program to convert the sheet music the user wanted to play into MIDI files. Though this project could have taken many forms as to what the user could input to specify the song they wanted to play, we settled upon sheet music for being a key method of musical notation. We also believe this could enhance Piano Press's functions due to the fact that some rheumatoid arthritis patients may have blurred or lost vision (Matsuo 2001), and thus Piano Press could serve as their third eye in music. We designed a user interface for how we plan to implement a front-end side to this program (in the form of an application), whose early screen sketches can be found in Figure 12.
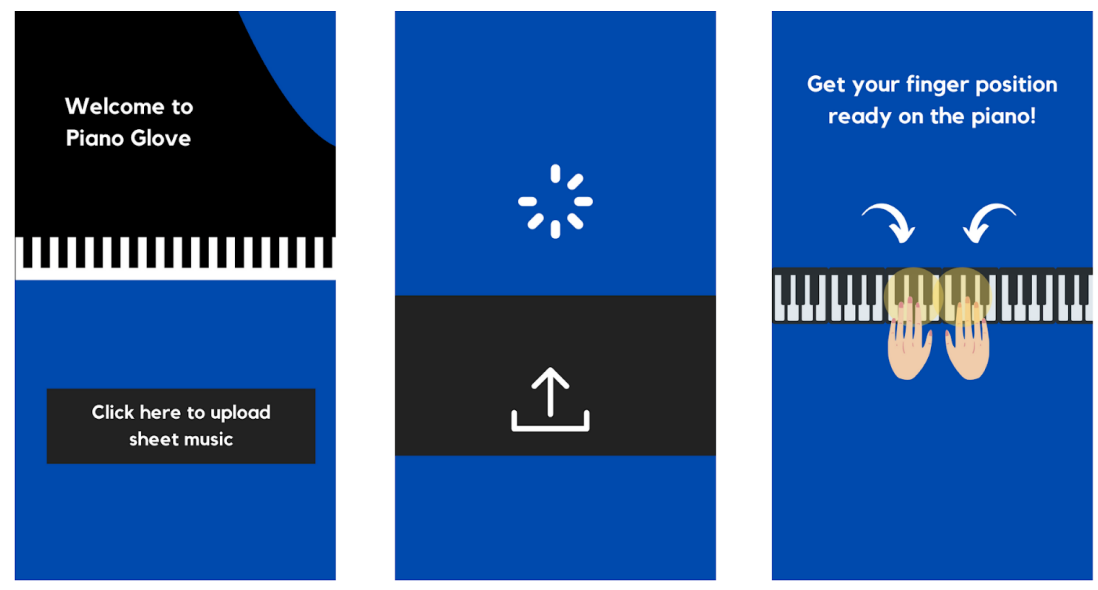
Figure 12: Our initial designs for a potential application that is compatible with our device. It would include a simple User Interface, an option to upload a single picture of sheet music, and where to place fingers for the starting positions.

The program we chose to perform the task of converting sheet music to MIDI files is Sheet Vision. Sheet Vision is a python program using a template matching algorithm and OpenCV (Open Computer Vision) to scan for notes, flats, and sharps to create an output MIDI file. Sheet Vision was made by Calvert Pratt, Calvin Gregory, and Alex Reichenbach and can be found on GitHub, while using python libraries from Christoph Gohlke at the University of California, Irvine. We selected this program due to its methodical approach with an outlined six-step process and fairly efficient code. Once an image of sheet music is inputted into the program in the form of an argument, the program goes through multiple templates of notes, flats, and sharps to filter out parts of the image. A few of these templates can be found in Figure 13. Sheet Vision then goes through steps of character classification, note identification and sequencing, and finally compiling all these results and exporting it to the desired MIDI file. We ran multiple tests using Sheet Vision with example sheet music as well as external sources of music found online. These tests are touched upon in further detail in our implemented, PianoPlayer.

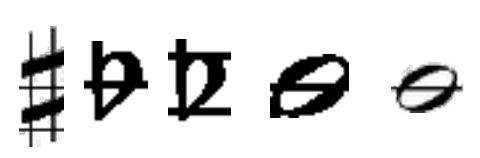
discussion of the second program we

Figure 13: Some of the templates provided by Sheet Vision to match to sheet music. Labels of images provided by Sheet Vision from left to right are, respectively, "f-sharp", "flat-line", "flat-space", "half-line", and "half-note-line". (Sheet Vision))

Though Sheet Vision allowed us to use a piece of sheet music the user wanted to play and outputted a MIDI file, this MIDI file alone could not tell us much as to how we should actuate each finger in Piano Press. We first approached this problem by extracting the notes Sheet Vision identified and creating conditional statements to correspond notes to fingers, but realized that multiple components go into the fingering of various songs, such as starting position of the fingers. Thus, we turned to PianoPlayer, a python program made by Marco Musy, Pedro, Philip Abbet, and Sebastian Hofstetter which can also be sourced from GitHub. This program factors in a variety of elements, and is described by the creators as "dynamic" due to its ability to account for physical positioning of the hands, the speed of the fingers playing the sound, and the duration of the note (PianoPlayer). PianoPlayer also searches for optimal finger combinations that would avoid any unnecessary movements of the hand, which is especially beneficial in our scenario because Piano Press's target audience would be arthritic patients who need to avoid the sometimes spontaneous movements in piano.

Due to issues with our accustomed IDE, we tested PianoPlayer in Mac's terminal. A simple input of "pianoplayer" without specifying any arguments outputs a GUI with four main options (Figure 14). Our first test was with the song "Hot Cross Buns", an elementary piano song with minimal sheet music. Once this sheet music went through Sheet Vision, it outputted a MIDI file and an analyzed image of the sheet music. The MIDI file was then inputted into PianoPlayer using "Import Score" and "Generate" to output a list of hand and finger combinations necessary to play the song. A 3D Player can also be pulled up to visualize the fingering on a piano. This process is illustrated in Figure 15. 


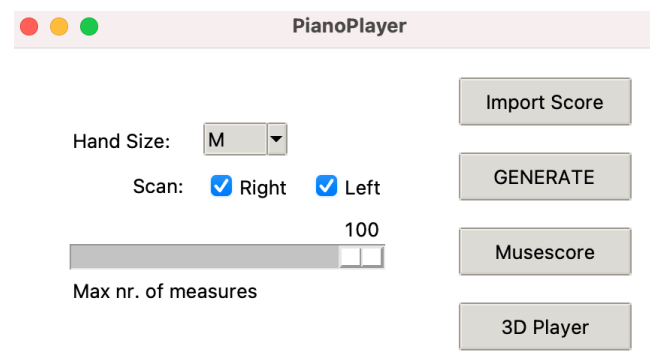

Figure 14: The Graphical User Interface (GUI) of PianoPlayer. Parameters can also be changed to alter the hand size which would then force the program to change certain fingering positions to maximize efficiency on the piano.
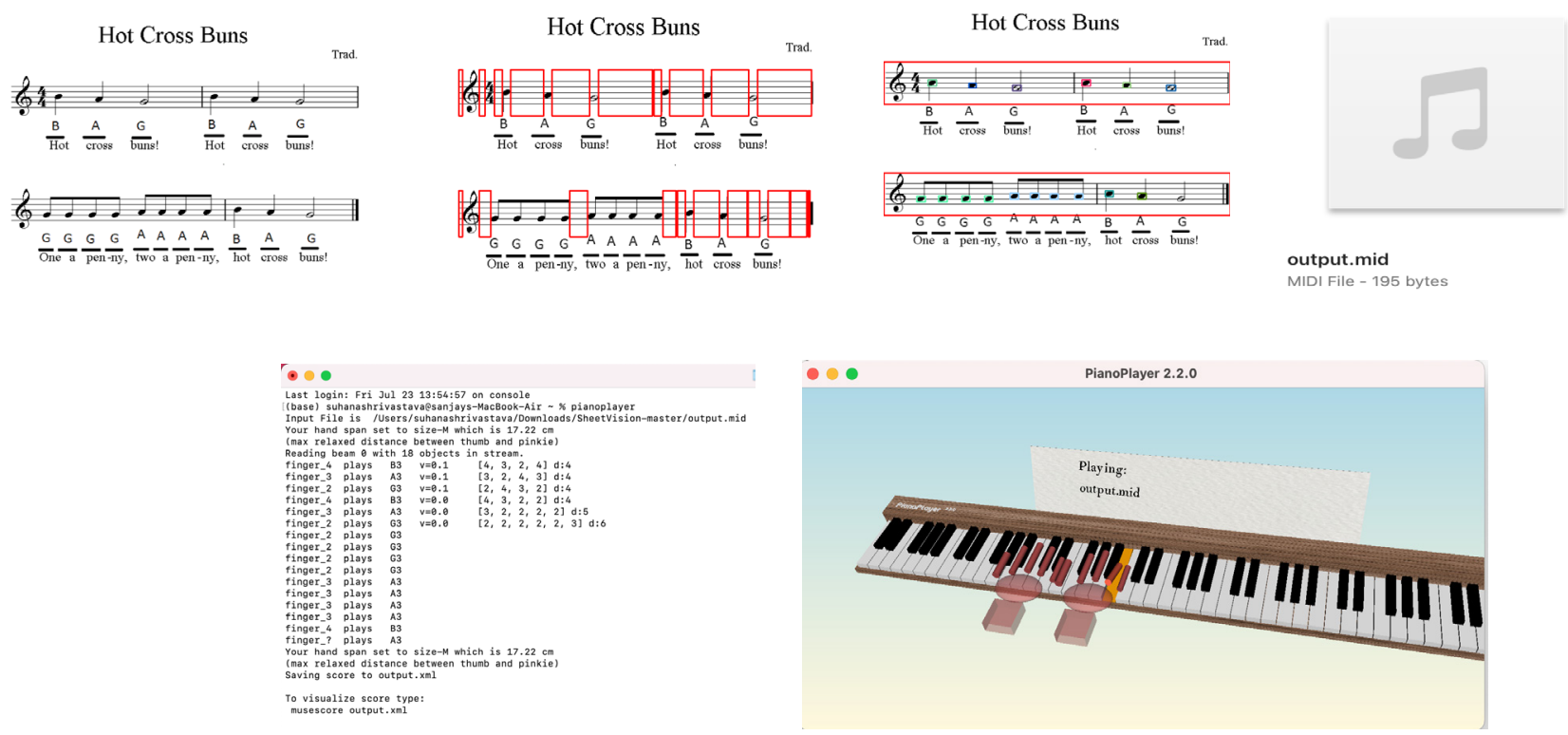

Figure 15: The top column identifies a few of the steps in analyzing the sheet music "Hot Cross Buns" including identifying thresholds and notes in Sheet Vision, and the MIDI file called output.mid. The second column shows PianoPlayer using the outputted MIDI file from Sheet Vision, with the figure on the left displaying the fingering of the hands and the figure on the right displaying the 3D representation. The original hot cross buns sheet music was found on (Sheet Vision, PianoPlayer).

\section{Results}

\section{Bend Angle Analysis}

To gauge the accuracy of the glove bending compared to a real pianist's hands, we decided to analyze two bending angles in the joints. Although the human finger has three joints, we only focused on the proximal interphalangeal joint (PIP) and the metacarpophalangeal joint (MCP), the two closest to the fingertip, because of limitations with the 3D model. The data we used were side images of the hand and glove models since they clearly showed the configuration of the joints. To accurately measure the bending angles, we imported those images into Solidworks and drew a skeleton structure over the real and model hands. Then the angle of each joint was measured and compared. We found that our model hand differed from the real hand $21.08 \%$ in Angle 1 and $15.08 \%$ in Angle 2. These values are quite off our 
target which is to have Piano Press exactly mimic the real hand model. A potential cause for this high inaccuracy is the limitations of our 3D model bending in Rhino using the Grasshopper parametric plugin. Since our CAD model does not bend based on the cable pulling, there is a considerable margin of error.

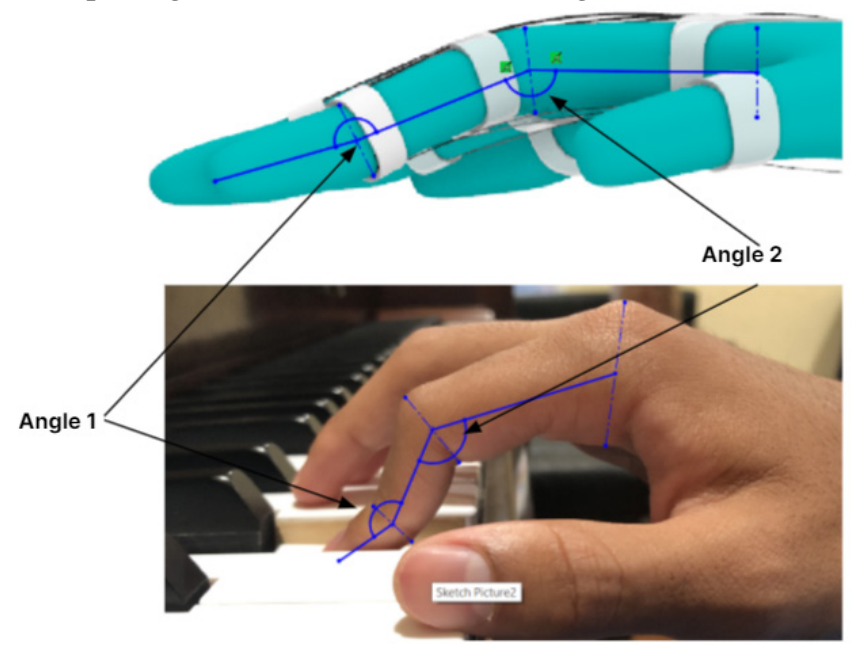

Figure 16: Real vs. model hand joint angle analysis

Table 1: Bend angle analysis data

\begin{tabular}{|l|l|l|l|}
\hline & Real Hand & Model Hand & $\begin{array}{l}\text { Model to Real Hand \% differ- } \\
\text { ence }\end{array}$ \\
\hline Angle 1 (degrees) & 157.33 & 129.94 & $21.08 \%$ \\
\hline Angle 2 (degrees) & 173.94 & 147.71 & $15.08 \%$ \\
\hline
\end{tabular}

\section{Discussion and Conclusion}

Piano Press presents a design inspired by other cable-actuated gloves that is the first compact, tetherless, and selfguiding glove with complete finger actuation. It builds upon past designs with a wearable hardware pack, utilizing algorithms to control finger movement based on sheet music, and having the ability to actuate all 10 fingers. Its three key modules: the software portion which allows us to take the sheet music the user wants to play and convert that into a finger combination sequence, the glove portion that was designed for user comfort and 3D modeled, and the hardware portion with a hardware pack and the microcontroller also differentiate it from past models. These three modules allow Piano Press to have a wide variety of applications, including improving upon the traditional methods of physical therapy, and even being employed by typers with modifications to the software portion.

The model to real hand difference achieved (21.08\% for angle 1 and $15.08 \%$ for angle 2$)$ is somewhat expected due to our limitations with Rhino, however it proves the need for improvements in this area. The real vs. model hand joint angle analysis presented in Figure 16 outlines the differences in the real hand compared to the model hand, and it can be seen that the real hand has greater ability to attain optimal curvature to hit the correct piano key. At its current state, Piano Press cannot determine the position of the user's fingers relative to the keyboard and thus could not be moved like the real hand could. To compensate for this in the future, we hope to test Piano Press in real life to gain more accurate angle measurements for our model hand and test them to determine if the pulling of cables can simulate the real hand or if design changes need to be made. 
Going into the future, we hope to build the necessary hardware and glove portions of the device for further testing and therapy application, and create a better front end that allows for simple, smooth interaction with the user. Overall, Piano Press has tremendous application for improving physical therapy in RA patients with its innovative design aspects and customizable music options.

\section{Limitations and Future Research}

Due to Covid-19 restrictions, our research was conducted fully online. Since we did not have access to the facilities at UCSB to build a physical prototype, our design and analysis of Piano Press was conducted through CAD models, image comparisons of the models, and software testing. Making a real prototype would include casting a silicone mold for the glove, 3D printing the hardware pack, wiring several electronic components and motors, routing the bowden cables to the fingers, and running our algorithm on Arduino C. For further research, we would like to create a physical prototype of Piano Press to get more accurate testing on real human hands. We would do a frame by frame video analysis of the Piano Press playing a simple song like "Hot Cross Buns," comparing that to a real pianist's demonstration of the song. This could involve analyzing the stiffness of the Piano Press and comparing it to a real pianist's fingers, as a weaker stiffness would result in a more inconsistent or incorrect sounding keypress.

\section{Acknowledgments}

We would like to thank our instructors Yin Yu, Diarmid Flatley, and Alanna Bartolini for providing us feedback and assisting us with our research. We also appreciate Dr. Sheila Jiang's advisory for our overall design and advice on how we can make Piano Press more arthritis-friendly. Additionally, we would like to thank Calvert Pratt, Calvin Gregory, and Alex Reichenbach for creating Sheet Vision and Marco Musy, Pedro, Philip Abbet, and Sebastian Hofstetter for PianoPlayer, the two python programs we used. We would also like to extend our gratitude to Dr. Kim and the Summer Research Academies (SRA) for hosting this research program.

\section{Author Contribution Statement}

S.S. carried out the software portions of the project, J.L. designed the 3D modeled glove, and V.P. created the hardware pack of Piano Press. All authors reviewed and contributed equally to the manuscript.

\section{References}

Cappello, L., Meyer, J.T., Galloway, K.C. et al. Assisting hand function after spinal cord injury with a fabric-based soft robotic glove. J NeuroEngineering Rehabil 15, 59 (2018). https://doi.org/10.1186/s12984-018-0391-x

Hughes, J A E, P. Maiolino, and F. Iida. "An Anthropomorphic Soft Skeleton Hand Exploiting Conditional Models for Piano Playing." Science Robotics 3.25 (2018): Science Robotics, 19 December 2018, Vol.3(25). Web.

In, H., Kang, B. B., Sin, M. K., \&amp; Cho, K.-J. (2015). Exo-Glove: A Wearable Robot for the Hand with a Soft Tendon Routing System. IEEE Robotics \&amp; Automation Magazine, 22(1), 97-105. https://doi.org/10.1109/mra.2014.2362863

K. Huang, E. Y. Do and T. Starner, "PianoTouch: A wearable haptic piano instruction system for passive learning of piano skills," 2008 12th IEEE International Symposium on Wearable Computers, 2008, pp. 41-44, doi: 10.1109/ISWC.2008.4911582.

Kinoshita, Hiroshi \& Furuya, Shinichi \& Aoki, Tomoko \& Nakahara, Hidehiro \& Altenmüller, Eckart. (2007). Characteristics of keystroke force in the piano. Journal of Biomechanics - J BIOMECH. 40. 
10.1016/S0021-9290(07)70392-5.

L. T. Estes, D. Backus and T. Starner, "A wearable vibration glove for improving hand sensation in persons with Spinal Cord Injury using Passive Haptic Rehabilitation," 2015 9th International Conference on Pervasive Computing Technologies for Healthcare (PervasiveHealth), 2015, pp. 37-44, doi: 10.4108/icst.pervasivehealth.2015.259137.

Li, Y.-F., \&amp; Lai, C.-Y. (2014). Intelligent algorithm for music playing robot — Applied to the anthropomorphic piano robot control. 2014 IEEE 23rd International Symposium on Industrial Electronics (ISIE). https://doi.org/10.1109/isie.2014.6864843

Matsuo, T. (2001, December 17). Multiple Occlusive Retinal Arteritis in Both Eyes of a Patient with Rheumatoid Arthritis. Japanese Journal of Ophthalmology. https://www.sciencedirect.com/science/article/pii/S0021515501004105.

Neun, John V. (2017). JVN Design Calculator. https://www.chiefdelphi.com/uploads/default/original/3X/2/b/2bf9206b962f74ed5556a0ae936ef0bf365ac9 75.xlsx

S. Sugano and I. Kato, "WABOT-2: Autonomous robot with dexterous finger-arm--Finger-arm coordination control in keyboard performance," Proceedings. 1987 IEEE International Conference on Robotics and Automation, 1987, pp. 90-97, doi: 10.1109/ROBOT.1987.1088025.

Smolen, J. S., Aletaha, D., \&amp; McInnes, I. B. (2016). Rheumatoid arthritis. The Lancet, 388(10055), $2023-2038$. https://doi.org/10.1016/s0140-6736(16)30173-8

Vandever, L. (2019, January 15). Rheumatoid Arthritis by the Numbers: Facts, Statistics, and You. Healthline. https://www.healthline.com/health/rheumatoid-arthritis/facts-statistics-infographic\#Prevalence.

Zhang, D., Lei, J., Li, B., Lau, D., \&amp; Cameron, C. (2009). Design and analysis of a piano playing robot. 2009 International Conference on Information and Automation. https://doi.org/10.1109/icinfa.2009.5205022

External links for our Python Programs:

https://github.com/cal-pratt/SheetVision

https://www.lfd.uci.edu/ gohlke/pythonlibs/ 